\title{
HIGHER INTEGRABILITY OF MINIMIZERS OF DEGENERATE FUNCTIONALS IN CARNOT-CARATHÉODORY SPACES
}

\section{Patrizia Di Gironimo and Flavia Giannetti}

Università degli Studi di Salerno, Dipartimento di Matematica

Via Giovanni Paolo II 132, 84084 Fisciano (SA), Italia; pdigironimo@unisa.it

Università degli Studi di Napoli, Dipartimento di Matematica e Applicazioni "R. Caccioppoli" Via Cintia, 80126 Napoli, Italia; giannett@unina.it

\footnotetext{
Abstract. In this paper, we prove a higher integrability result for the horizontal gradient of a minimizer of a functional of the type

$$
I(\Omega, u)=\int_{\Omega} \sum_{i, j} a_{i, j} X_{i} u X_{j} u d x
$$

whose matrix of the coefficients $A(x)={ }^{t} A(x)$ satisfies the anisotropic bounds

$$
\frac{|\xi|^{2}}{K(x)} \leq\langle A(x) \xi, \xi\rangle \leq K(x)|\xi|^{2} \quad \forall \xi \in \mathbf{R}^{n}, \text { for a.e. } x \in \Omega,
$$

where the ellipticity function $K(x) \in A_{2} \cap R H_{\tau}, \tau$ opportunely related to the homogeneous dimension, and is such that the pair $\left(K, \frac{1}{K}\right) \in A_{1}$.
}

\section{Introduction}

Let $\Omega$ be a bounded open set in $\mathbf{R}^{n}$ and $X=\left(X_{1}, \ldots, X_{k}\right)$ be a family of vector fields defined in a neighborhood of $\Omega$, with real, $C^{\infty}$ smooth and globally Lipschitz coefficients satisfying the Hörmander condition.

Let us consider the following functional

$$
I(\Omega, u)=\int_{\Omega} \sum_{i, j=1}^{k} a_{i, j} X_{i} u X_{j} u d x
$$

where the matrix of the coefficients $A(x)=\left(a_{i j}(x)\right)_{i, j}$ is symmetric and satisfies the following anisotropic bounds

$$
\frac{|\xi|^{2}}{K(x)} \leq\langle A(x) \xi, \xi\rangle \leq K(x)|\xi|^{2} \quad \forall \xi \in \mathbf{R}^{n}, \text { for a.e. } x \in \Omega
$$

with

$$
K: \Omega \subset \mathbf{R}^{n} \rightarrow[1,+\infty), \quad K \in L_{\mathrm{loc}}^{1}(\Omega) .
$$

We suppose that the function $K$ is a weight belonging to the class $A_{2} \cap R H_{\tau}$, with $\tau$ related to the homogeneous dimension, and is such that the pair $\left(K, \frac{1}{K}\right) \in A_{1}$ (see Section 2 for the definitions). Recalling that the Sobolev space $W_{X}^{1, p}(\Omega)$ is defined as

$$
W_{X}^{1, p}(\Omega)=\left\{u \in L^{p}(\Omega): X_{j} u \in L^{p}(\Omega), j=1, \ldots, k\right\},
$$


we denote by $H_{X}(\Omega)$ the set of all $u \in W_{X, \text { loc }}^{1,1}(\Omega)$ such that $u \in L^{2}(\Omega, K d x)$ and $u$ has finite energy, i.e. verifies

$$
\langle A X u, X u\rangle \in L^{1}(\Omega) .
$$

Clearly, $H_{X}(\Omega)$ is a vector space and, since $\Omega$ is bounded, we can make it a normed vector space by taking the norm induced by the bilinear form

$$
\alpha(u, \varphi)=\int_{\Omega}\langle A X u(x), X \varphi(x)\rangle d x+\int_{\Omega} u(x) \varphi(x) K(x) d x .
$$

Define $H_{X, L}(\Omega)$ as the Hilbert space obtained by taking the closure of Lipschitz functions with respect to this norm. To be precise, the elements of $H_{X, L}(\Omega)$ are Cauchy sequences $\left\{u_{k}\right\}$ of Lipschitz functions. It follows from the definition (1.5) and condition (1.2) that $u_{k}$ converges in $L^{2}(\Omega, K d x)$ and $X u_{k}$ converges in $L^{2}\left(\Omega, \frac{1}{K} d x\right)$. However, we do not know a priori whether an arbitrary $u \in H_{X}(\Omega)$ is in $H_{X, L}(\Omega)$ unless we make additional assumptions on $u$, such as assuming $X u \in L^{2}\left(\Omega, \frac{1}{K} d x\right)$. Nevertheless, hereafter, when we write $u \in H_{X, L}(\Omega)$ we mean that $u$ is actually a function in $H_{X}(\Omega) \cap H_{X, L}(\Omega)$ and not a Cauchy sequence.

Recall the following

Definition 1.1. A function $u \in H_{X}(\Omega)$ is a local minimizer for $I(\Omega, u)$ if

$$
I(\operatorname{supp} \varphi, u) \leq I(\operatorname{supp} \varphi, u+\varphi)
$$

for any $\varphi \in H_{X}(\Omega)$ with compact support.

The aim of this paper is to study the regularity of the local minimizers of $I(\Omega, u)$ which generalizes functionals of the type

$$
\mathcal{F}(\Omega, u)=\int_{\Omega} \sum_{i, j=1}^{k} a_{i, j} D_{i} u D_{j} u d x .
$$

More precisely we shall prove the following higher integrability result:

Theorem 1.1. Let $u \in H_{X, L}(\Omega)$ be a local minimizer for the functional (1.1) with the matrix of the coefficients satisfying (1.2). Suppose $K \in A_{2}(\Omega, \rho) \bigcap R H_{\tau}(\Omega, \rho)$ with $\tau=1+\frac{2(Q-1)}{n+2-Q}$ and such that the pair $\left(K, \frac{1}{K}\right) \in A_{1}(\Omega, \rho)$. Then $X u \in$ $L_{\text {loc }}^{p}\left(\Omega, \frac{1}{K} d x\right)$ for some $p>2$ and there exists a constant $C$ such that, for each ball $B_{R} \subset \subset \Omega$, it holds

$$
\left(f_{B_{\frac{R}{2}}}|X u|^{p} d \nu\right)^{\frac{1}{p}} \leq C\left(f_{B_{R}}|X u|^{2} d \nu\right)^{\frac{1}{2}}
$$

where $d \nu=\frac{1}{K} d x$.

We point out that the tools used to obtain the higher integrability are the classical ones of the euclidean case. Neverthless, here we deal with functionals whose integrands have a degeneracy in the coefficients expressed by mean of the assumption (1.2) and, at the same time, a double degeneracy into the geometry due to the presence of a differential operator different from the classical gradient. In particular, this second peculiarity causes a change of metric in $\mathbf{R}^{n}$ and therefore the use of results valid for arbitrary metric spaces. 
The study of the regularity of local minimizers of functionals depending on vector fields of the type

$$
\int_{\Omega} f(X u) d x
$$

as far as we know, has been not largely studied. It seems to us that the literature available on the regularity of solutions of equations is more vast (see for example $[17,3,7,5]$ and references therein).

Anyway, the regularity of local minimizers of integral functionals is interesting by itself since the passage to the Euler-Lagrange equation is not always possible. For example, in [12], assuming that the integrand grows as $|\xi|^{p}$ for some $p>1$, and satisfies the following Lipschitz assumption

$$
|f(\xi)-f(\eta)| \leq \beta|\xi-\eta|^{p}\left(|\xi|^{p-1}+|\xi-\eta|^{p-1}\right),
$$

it has been proven that any weak minimizer of (1.7) is actually a local minimizer. Later on, in [13], assuming $f$ satisfying the non-standard growth condition

$$
c_{1}(A(|\xi|)-1) \leq f(\xi) \leq c_{2}(A(|\xi|)+1)
$$

with $A$ suitable $N$-function, a regularity result in the scale of Campanato spaces for local minimizers has been obtained, as well as an higher integrability result for their horizontal gradients.

We also indicate [11] for higher integrability results for quasi-minima of functionals with standard and non-standard growth conditions like

$$
c_{1}|\xi|^{p} \leq f(\xi) \leq c_{2}|\xi|^{q}
$$

with exponents $1<p \leq q$ opportunely related.

Finally, some results of local Hölder continuity in the context of a metric measure space where the measure is doubling and the space supports a weak Poincaré inequality, also in the case the integrand satisfies the non-standard growth condition above are available (see $[16,1,18]$ ).

Concerning the case of variational integrals, whose integrands have a degeneracy expressed through Muckenhoupt weights, we mention the pioneering paper by Modica [19] but also the contribution given by Moscariello in [20]. Anyway, they both concern the euclidean setting and not a metric context.

Actually, it is worth pointing out that in [5], the authors give a result of local Hölder continuity for the weak solutions of the $p$-Laplace equation

$$
\operatorname{div}\left(\langle A X u, X u\rangle^{\frac{p-2}{2}} A X u\right)=0
$$

where the ellipticity function is of the type considered in Theorem 1.1 (see also [6] for the euclidean setting).

\section{Notation and preliminary results}

In order to frame the setting of our problem, we devote this section to some useful notation and preliminaries. First of all, we recall the following iteration lemma (see Lemma 1.1 in [14]):

Lemma 2.1. Let $f(t)$ be a nonnegative bounded function defined for $0 \leq T_{0} \leq$ $t \leq T_{1}$. Suppose that for $T_{0} \leq t<s \leq T_{1}$,

$$
f(t) \leq A(s-t)^{-\gamma}+B+\theta f(s),
$$


where $A, B, \gamma, \theta$ are nonnegative constants and $\theta<1$. Then there exists a constant $c$, depending only on $\gamma$ and $\theta$ such that for every $\beta, R, T_{0} \leq \beta<R \leq T_{1}$, one has

$$
f(\beta) \leq c\left[A(R-\beta)^{-\gamma}+B\right]
$$

2.1. Carnot-Carathéodory spaces. Let $X=\left(X_{1}, \cdots, X_{k}\right)$ be a family of vector fields defined in $\mathbf{R}^{n}$, with real,$C^{\infty}$ smooth coefficients. We say that they satisfy the Hörmander's condition if there exists an integer $m$ such that the family of commutators of $X_{1}, \cdots, X_{k}$ up to length $m$

$$
\left.X_{1}, \cdots, X_{k},\left[X_{i_{1}}, X_{i_{2}}\right], \cdots,\left[X_{i_{1}},\left[X_{i_{1}}, \cdots X_{i_{m}}\right]\right] \cdots\right], \quad \forall i_{j}=1,2, \cdots, k
$$

spans the tangent space $T_{x} \mathbf{R}^{n}$ at every point $x \in \mathbf{R}^{n}$.

For any real valued Lipschitz continuous function $u$ we define

$$
X_{j} u(x)=\left\langle X_{j}(x), \nabla u(x)\right\rangle, \quad j=1,2, \cdots, k,
$$

and we call the horizontal gradient of $u$ the vector $X u=\left(X_{1} u, \cdots, X_{k} u\right)$ whose length is given by

$$
|X u|=\left(\sum_{j=1}^{k}\left(X_{j} u\right)^{2}\right)^{1 / 2} .
$$

An absolutely continuous curve $\gamma:[a, b] \rightarrow \mathbf{R}^{n}$ is said to be admissible, if there exist functions $c_{j}:[a, b] \rightarrow \mathbf{R}, j=1, \cdots, k$, such that

$$
\dot{\gamma}(t)=\sum_{j=1}^{k} c_{j}(t) X_{j}(\gamma(t)) \quad \text { and } \quad \sum_{j=1}^{k} c_{j}(t)^{2} \leq 1 .
$$

Observe that $X_{j}$ do not need to be linearly independent and therefore functions $c_{j}$ do not need to be unique. Define the distance function $\rho$ as

$$
\rho(x, y)=\inf \left\{T>0: \exists \gamma:[0, T] \rightarrow \mathbf{R}^{n} \text { admissible, } \gamma(0)=x, \gamma(T)=y\right\} .
$$

If there is not any such a curve, we set $\rho(x, y)=\infty$. The function $\rho$ is called CarnotCarathéodory distance and, since it is not clear whether one can connect any two points of $\mathbf{R}^{n}$ by an admissible curve, it's not clear whether $\rho$ is a metric.

The assumption for which the vector fields $X_{1}, \cdots, X_{k}$ satisfy the Hörmander condition ensures that $\rho$ is a metric and in this case $\left(\mathbf{R}^{n}, \rho\right)$ is said to be a CarnotCarathéodory space.

The following theorem, due to Nagel, Stein and Wainger [21], shows that the metric $\rho$ is locally Hölder continuous with respect to the Euclidean metric.

Theorem 2.2. Let $X_{1}, \cdots, X_{k}$ be as above. Then for every bounded open set $\Omega \subset \mathbf{R}^{n}$ there are constants $c_{1}, c_{2}$ and $\lambda \in(0,1]$ such that

$$
c_{1}|x-y| \leq \rho(x, y) \leq c_{2}|x-y|^{\lambda}
$$

for every $x, y \in \Omega$.

It follows that the space $\left(\mathbf{R}^{n}, \rho\right)$ is homeomorphic with the Euclidean space $\mathbf{R}^{n}$ and therefore bounded sets in the Euclidean metric are bounded sets in the metric $\rho$. The inverse is not always true but it is certainly valid if $X_{1}, \cdots X_{k}$ have globally Lipschitz coefficients (see [10]). In the sequel all the distances will be respect to the metric $\rho$, in particular all the balls will be balls with respect to the CarnotCarathéodory metric. We shall consider in $\left(\mathbf{R}^{n}, \rho\right)$ the Lebesgue measure $|\cdot|$ and 
denote by $f_{B} f(x) d x$ the average of the function $f$ on the ball $B$, i.e.

$$
f_{B} f(x) d x=\frac{1}{|B|} \int_{B} f(x) d x
$$

Note that the Lebesgue measure locally satisfies the following doubling condition (see for example [21]):

Proposition 2.3. Let $\Omega$ be an open, bounded subset of $\mathbf{R}^{n}$. There exists a constant $C_{d} \geq 1$, called doubling constant, such that

$$
\left|B\left(x_{0}, 2 R\right)\right| \leq C_{d}\left|B\left(x_{0}, R\right)\right|
$$

provided the center $x_{0} \in \Omega$ and the radius $R \leq 5 \operatorname{diam} \Omega$.

Let $Y$ be a metric space and $\mu$ a Borel measure in $Y$. Assume $\mu$ finite on bounded sets and satisfying the doubling condition on every open, bounded subset $\Omega$ of $Y$. If there exists a positive constant $C$ such that

$$
\frac{\mu(B)}{\mu\left(B_{0}\right)} \geq C\left(\frac{R}{R_{0}}\right)^{Q}
$$

for any ball $B_{0}$ having center in $\Omega$ and radius $R_{0}<\operatorname{diam} \Omega$ and any ball $B$ centered in $x_{0} \in B_{0}$ and having radius $R \leq R_{0}$, we say that $Q$ is a homogeneous dimension relative to $\Omega$.

It is well known that the doubling condition implies the existence of such a $Q$. However, $Q$ is not unique and it may change with $\Omega$. Obviously any $Q^{\prime} \geq Q$ is also a homogeneous dimension. For a bounded open set $\Omega$ containing a family of vector fields satisfying the Hörmander condition, the Carnot-Carathéodory space $(\Omega, \rho)$ with the Lebesgue measure has the homogeneous dimension $Q=\log _{2} C_{d}$.

We shall need the following version of Gehring Lemma for metric spaces with a doubling measure $\mu$.

Lemma 2.4. [11, 24] Let $q_{0} \leq q \leq 2 Q$ where $q_{0}>1$ is fixed. Assume $f \in$ $L_{\mathrm{loc}}^{r_{0}}(Y, \mu)$ and $g \in L_{\mathrm{loc}}^{q}(Y, \mu), r_{0}>q$, be nonnegative functions and suppose the existence of constants $b>1$ and $0<\vartheta<1$ such that for every ball $B \subset \sigma B \subset Y$, the following inequality holds

$$
f_{B} g^{q} d \mu \leq b\left[\left(f_{\sigma B} g d \mu\right)^{q}+f_{\sigma B} f^{q} d \mu\right]+\vartheta f_{\sigma B} g^{q} d \mu .
$$

Then there exist nonnegative constants $\theta_{0}=\theta_{0}\left(q_{0}, Q, C_{d}, \sigma\right)$ and $\varepsilon_{0}=\varepsilon_{0}\left(b, q_{0}, Q, C_{d}, \sigma\right)$ such that, if $0 \leq \vartheta<\theta_{0}$, then $g \in L_{\mathrm{loc}}^{p}(Y, \mu)$ for $q \leq p<q+\varepsilon_{0}$ and moreover

$$
\left(f_{B} g^{p} d \mu\right)^{1 / p} \leq C\left[\left(f_{\sigma B} g^{q} d \mu\right)^{1 / q}+\left(f_{\sigma B} f^{q} d \mu\right)^{1 / p}\right]
$$

for $C=C\left(b, q_{0}, Q, C_{d}, \sigma\right)$.

Note that we used the notation $\sigma B$ for the ball concentric with $B$ having radius $\sigma$-times that of $B$.

2.2. Muckenhoupt weights. In the following we recall some useful definitions and properties; we refer to [23] or [22] for more details.

Let $\Omega \subset \mathbf{R}^{n}$ be an open, bounded and connected set. By a weight we mean a positive function in $L_{\text {loc }}^{1}\left(\mathbf{R}^{n}\right)$. We say that a weight $w$ is doubling in $\Omega$ if

$$
\int_{2 B} w(x) d x \leq C \int_{B} w(x) d x
$$


where the constant $C$ is independent by the ball $B \subset \Omega$. We say that $w$ is locally doubling in $\Omega$ if for each compact set $V \subset \Omega$ and $R>0$ there exists $C_{V, R}$ such that

$$
\int_{2 B} w(x) d x \leq C_{V, R} \int_{B} w(x) d x
$$

where the ball $B$ has center in $V$ and radius $r<R$. Given $1<p<\infty$ and a weight $w$, we say $w \in A_{p}(\Omega, \rho)$ if

$$
[w]_{A_{p}}:=\sup _{B}\left(f_{B} w(x) d x\right)\left(f_{B} w(x)^{1-p^{\prime}} d x\right)^{p-1}<\infty
$$

where the supremum is taken over all balls $B \subset \Omega$ and $p^{\prime}$ denotes the Hölder conjugate exponent of $p$. When $p=1$, we say that $w \in A_{1}(\Omega, \rho)$ if there exists a constant $c \geq 1$ such that, for every ball $B \subset \Omega$, it holds

$$
\int_{B} w(x) d x \leq c \operatorname{ess} \inf _{B} w
$$

Weights belonging to a class $A_{p}$ are called Muckenhoupt weights. Moreover, we say that $w$ satisfies a reverse Hölder inequality in $\Omega$ with exponent $t>1$, and we write $w \in R H_{t}(\Omega, \rho)$, if

$$
[w]_{R H_{t}}:=\sup _{B} \frac{\left(f_{B} w(x)^{t} d x\right)^{\frac{1}{t}}}{f_{B} w(x) d x}<\infty .
$$

Next two Lemmas contain useful properties for the weights defined above.

Lemma 2.5. If $w \in A_{p}(\Omega, \rho)$ for some $p>1$, then

1) $w^{1-p^{\prime}} \in A_{p^{\prime}}(\Omega, \rho)$;

2) $\exists t>1$ such that $w \in R H_{t}(\Omega, \rho)$;

3) $\exists \varepsilon>0$ such that $w \in A_{p-\varepsilon}(\Omega, \rho)$.

As in the Euclidean case, assertion 1) is a direct consequence of the definition of $A_{p}$ weight; assertion 2) is proved in [22] and assertion 3) easily follows from 1) and $2)$.

Lemma 2.6. 1$)$ If $w \in R H_{t}(\Omega, \rho)$ for some $t>1$, then for any $x_{0} \in \Omega$, $r>0$ and any measurable set $E \subset B\left(x_{0}, r\right) \cap \Omega$, we have

$$
\frac{\int_{E} w(x) d x}{\int_{B\left(x_{0}, 2 r\right) \cap \Omega} w(x) d x} \leq[w]_{R H_{t}(\Omega, \rho)}\left(\frac{|E|}{\left|B\left(x_{0}, r\right) \cap \Omega\right|}\right)^{\frac{1}{t^{\prime}}} .
$$

2) If $w \in A_{p}(\Omega, \rho)$ for some $p>1$, then for any $x_{0} \in \Omega, r>0$ and any measurable set $E \subset B\left(x_{0}, r\right) \cap \Omega$, it holds

$$
\frac{|E|}{\left|B\left(x_{0}, r\right) \cap \Omega\right|} \leq\left([w]_{R H_{t}(\Omega, \rho)} \frac{\int_{E} w(x) d x}{\int_{B\left(x_{0}, 2 r\right) \cap \Omega} w(x) d x}\right)^{\frac{1}{p}}
$$

and

$$
\int_{B\left(x_{0}, 2 r\right) \cap \Omega} w(x) d x \leq C_{d}^{p}[w]_{A_{p}} \int_{B\left(x_{0}, r\right) \cap \Omega} w(x) d x,
$$

where $C_{d}$ is a doubling constant for $(\Omega, \rho)$, that is $w$ is doubling. 
The proof of previous Lemma can be found in [9] in the euclidean setting, we suggest [5] for some comments useful in our context.

Finally, we recall that a pair of weights $(w, v) \in A_{1}(\Omega, \rho)$ provided

$$
f_{B} w(x) d x \leq c \operatorname{ess} \inf _{B} v \text { for all } B \subset \Omega \text {. }
$$

In the sequel, we will be interested in the following class of weights, already introduced in [8].

Definition 2.7. Given a pair of weights $(w, v), w \leq v$ and an exponent $1<p<$ $\infty$, we say that the pair is $p$-admissible in $\Omega$ if

1) $v$ is locally doubling in $(\Omega, \rho)$ and $w \in A_{p}(\Omega, \rho)$;

2) for a given compact set $V \subset \Omega$ there exist $q>p$ and $C \geq 1$ such that, for every ball $B$ centered in $V$ and $0<r<1$, it holds

$$
r\left(\frac{\int_{r B} v(x) d x}{\int_{B} v(x) d x}\right)^{\frac{1}{q}} \leq C\left(\frac{\int_{r B} w(x) d x}{\int_{B} w(x) d x}\right)^{\frac{1}{p}} .
$$

This class of weights are fundamental for our aims since their properties imply the following two-weights Poincaré inequality (see [4] for $X=\nabla$ and [8] for the general case). We point out that the definition of $p$-admissible weight was given in [15].

Theorem 2.8. Let $1<p<\infty$ and $(w, v)$ be a pair of $p$-admissible weights in $\Omega$ and consider a compact set $V \subset \Omega$. Then there exists $R_{0}>0$ (depending on $V$ and $X)$ such that, if $B_{R}$ is a ball centered in $V$ and having radius $R<R_{0}$, then for all $u \in H_{X, L}\left(B_{R}\right)$ it holds

$$
\left(\frac{1}{\int_{B_{R}} v(x) d x} \int_{B_{R}}\left|u(x)-u_{R, v}\right|^{q} v d x\right)^{\frac{1}{q}} \leq C \cdot R\left(\frac{1}{\int_{B_{R}} w(x) d x} \int_{B_{R}}|X u(x)|^{p} w d x\right)^{\frac{1}{p}}
$$

where $C$ depends on $V, \Omega, X, u_{R, v}=\frac{1}{v\left(B_{R}\right)} \int_{B_{R}} u(x) v(x) d x$ and the constants in the Definition 2.7.

According to Definition 2.7, we give the following

Definition 2.9. We say that the measurable function $K \in L_{\mathrm{loc}}^{1}(\Omega)$ is $p_{0^{-}}$admissible if there exists $1<p_{0}<2$ and $C \geq 1$ such that for every ball $B$ and $0<r<1$,

$$
r\left(\frac{\int_{r B} K(x) d x}{\int_{B} K(x) d x}\right)^{\frac{1}{2}} \leq C\left(\frac{\int_{r B} K(x)^{-1} d x}{\int_{B} K(x)^{-1} d x}\right)^{\frac{1}{p_{0}}} .
$$

Sometimes $(2.12)$ will be referred to as the Chanillo-Wheeden condition with exponent $1<p_{0}<2$ and constant $C \geq 1$.

The following sufficient condition for locally integrable functions to be $p_{0}$-admissible is analogous to Theorem 4.8 in [5] (see also Proposition 4 in [6] ). For the reader's convenience we give the proof below.

Proposition 2.10. Let $2>Q-n$. The function $K$ is $p_{0}$-admissible provided $K^{-1} \in A_{p}(\Omega, \rho)$ and $K \in R H_{t}(\Omega, \rho)$ with $1<t<\infty$ and $\frac{2}{Q}<p<\infty$ satisfying

$$
\frac{2 t^{\prime}}{n} \leq\left(\frac{p Q}{p_{0}}\right)^{\prime}-1
$$

In particular, this is the case if $K \in A_{2}(\Omega, \rho) \cap R H_{\tau}(\Omega, \rho), \tau=1+\frac{2(Q-1)}{n+2-Q}$ 
Proof. Condition (2.13) is equivalent to

$$
\frac{n}{2 t^{\prime}} \geq \frac{p Q}{p_{0}}-1
$$

and the assumption $\frac{1}{K} \in A_{p}$ implies, by virtue of Lemma 2.5, the existence of $\varepsilon>0$ such that $\frac{1}{K} \in A_{p-\varepsilon}$. Obviously we get

$$
\frac{n}{2 t^{\prime}} \geq \frac{p Q}{p_{0}}-1>\frac{(p-\varepsilon) Q}{p_{0}}-1
$$

which implies

$$
\frac{n\left(t^{\prime}\right)^{-1}}{2}>(p-\varepsilon) Q p_{0}^{-1}-1
$$

Setting

$$
q=\frac{n\left(t^{\prime}\right)^{-1}}{(p-\varepsilon) Q p_{0}^{-1}-1},
$$

we deduce that $q>2$. Then, from the first assertion of Lemma 2.6 , we have for $0<r<1$ that

$$
r\left(\frac{\int_{r B} K(x) d x}{\int_{B} K(x) d x}\right)^{\frac{1}{q}} \leq c r\left(\frac{|r B|}{|B|}\right)^{\frac{1}{q t^{t}}}
$$

and therefore that

$$
r\left(\frac{\int_{r B} K(x) d x}{\int_{B} K(x) d x}\right)^{\frac{1}{q}} \leq c r^{\frac{n}{q t^{t}}+1}=C r^{\frac{(p-\varepsilon) Q}{p_{0}}} .
$$

Now, taking into account that the Lebesgue measure satisfies (2.2) and applying the second assertion of Lemma 2.6 , we have

$$
r\left(\frac{\int_{r B} K(x) d x}{\int_{B} K(x) d x}\right)^{\frac{1}{q}} \leq C\left(\frac{\int_{r B} K(x)^{-1} d x}{\int_{B} K(x)^{-1} d x}\right)^{\frac{p-\varepsilon}{p_{0} p}} \leq C\left(\frac{\int_{r B} K(x)^{-1} d x}{\int_{B} K(x)^{-1} d x}\right)^{\frac{1}{p_{0}}}
$$

since $\varepsilon$ is arbitrarily small. Since it is true for every ball $B$ and $q>2$, we deduce that $K$ is $p_{0}$-admissible.

\section{A two-weights Caccioppoli inequality and the main result}

For $K$ satisfying (1.3), it is legitimate to consider the measures

$$
d \nu=\frac{1}{K(x)} d x, \quad d \mu=K(x) d x
$$

and the Banach spaces $L^{p}(\Omega, d \nu), L^{p}(\Omega, d \mu)$ of all $p$-integrable functions with respect to the measures $d \nu$ and $d \mu$ equipped with the norms

$$
\|u\|_{p, \Omega, d \nu}=\left(\int_{\Omega}|u|^{p} d \nu\right)^{\frac{1}{p}}, \quad\|u\|_{p, \Omega, d \mu}=\left(\int_{\Omega}|u|^{p} d \mu\right)^{\frac{1}{p}} .
$$

In the next theorem we prove a two-weights Caccioppoli inequality. We point out that we don't need to assume any condition on the weight $K$.

Theorem 3.1. If $u \in H_{X}(\Omega)$ is a local minimizer for $I(\Omega, u)$ and (1.2) holds with $K$ satisfying (1.3) then, for any ball $B_{R}=B\left(x_{0}, R\right) \subset \Omega$ we have

$$
\int_{B_{\frac{R}{2}}}|X u|^{2} d \nu \leq C \int_{B_{R}} \frac{\left|u-u_{R}\right|^{2}}{R^{2}} d \mu .
$$


Proof. Let $t, s$ such that $\frac{R}{2}<t<s<R$ and define $\eta(x)=\psi\left(\left|x-x_{0}\right|\right)$, where $\psi \in C_{0}^{\infty}\left(B_{s}\right)$ is a cut-off function such that $\psi \equiv 1$ on $B_{t},|X \psi| \leq \frac{c}{s-t}$. The proof of the existence of a such function can be found, for example, in Lemma 3.6 of [10].

Observe that $\eta \in W_{X, 0}^{1, \infty}\left(B_{s}\right)$, the closure of $C_{0}^{\infty}$ in $W_{X}^{1, \infty}, \eta(x)=1$ on $B_{t}$ and

$$
|X \eta(x)|= \begin{cases}(s-t)^{-1}, & t \leq\left|x-x_{0}\right| \leq s \\ 0, & \text { otherwise. }\end{cases}
$$

Consider the function

$$
v(x)=u(x)+\eta^{2}(x)\left(u_{R, \mu}-u(x)\right) .
$$

For $x \in B_{s}$, observe that we have

$$
X v(x)=\left(1-\eta^{2}(x)\right) X u(x)+2 \eta^{2}(x) \frac{X \eta(x)}{\eta(x)}\left(u_{R, \mu}-u(x)\right) .
$$

Since $u$ is a minimizer and (1.2) holds, we have

$$
\begin{aligned}
& \int_{B_{s}}\langle A X u, X u\rangle d x \leq \int_{B_{s}}\langle A X v, X v\rangle d x \\
& =\int_{B_{s}}\left(1-\eta^{2}\right)\langle A X u, X u\rangle d x+\int_{B_{s}} 2 \eta^{2}\left\langle A\left(\frac{X \eta}{\eta}\left(u_{R, \mu}-u\right)\right), \frac{X \eta}{\eta}\left(u_{R, \mu}-u\right)\right\rangle d x \\
& \leq \int_{B_{s} \backslash B_{t}}\left(1-\eta^{2}\right)\langle A X u, X u\rangle d x+c \int_{B_{s}} \eta^{2} \frac{X^{2} \eta}{\eta^{2}}\left(u-u_{R, \mu}\right)^{2} K(x) d x .
\end{aligned}
$$

Then, by (3.2), we deduce that

$$
2 \int_{B_{t}}\langle A X u, X u\rangle d x \leq \int_{B_{s}}\langle A X u, X u\rangle d x+c \frac{1}{(s-t)^{2}} \int_{B_{s}}\left(u-u_{R, \mu}\right)^{2} K(x) d x .
$$

The assertion follows dividing for two both sides of last inequality and applying Lemma 2.1 and assumption (1.2).

Now we are in position to prove our main result on the regularity of minimizers.

Proof of Theorem 1.1. Let $B_{R}=B\left(x_{0}, R\right) \subset \Omega$. From Theorem 3.1 we immediately have that

$$
\int_{B_{\frac{R}{2}}}|X u|^{2} d \nu \leq C \int_{B_{R}} \frac{\left|u-u_{R, \mu}\right|^{2}}{R^{2}} d \mu .
$$

Observe now that assuming $K \in A_{2}$, by the second assertion of Lemma 2.6, we have that $K$ is doubling.

On the other hand, the assumption $K \in A_{2} \cap R H_{\tau}, \tau=1+\frac{2(Q-1)}{n+2-Q}$ implies, thanks to Proposition 2.10, that $K$ is $p_{0}$-admissible, where $p_{0}$ is related to $\tau$ by mean of (2.13), that is

$$
\frac{2 \tau^{\prime}}{n} \leq\left(\frac{2 Q}{p_{0}}\right)^{\prime}-1
$$

Therefore we have

$$
r\left(\frac{\int_{r B} K(x) d x}{\int_{B} K(x) d x}\right)^{\frac{1}{2}} \leq C\left(\frac{\int_{r B} K(x)^{-1} d x}{\int_{B} K(x)^{-1} d x}\right)^{\frac{1}{p_{0}}}
$$

It follows that the pair $\left(K^{-1}, K\right)$ verifies the two conditions of Definition 2.7 and hence is a pair of weights $p_{0}$-admissible. 
At this point we are legitimate to apply Theorem 2.8 having

$$
\left(\frac{1}{\int_{B_{R}} K(x) d x} \int_{B_{R}}\left|u(x)-u_{R, \mu}\right|^{2} d \mu\right)^{\frac{1}{2}} \leq C \cdot R\left(\frac{1}{\int_{B_{R}} K^{-1}(x) d x} \int_{B_{R}}|X u(x)|^{p_{0}} d \nu\right)^{\frac{1}{p_{0}}} .
$$

Combining estimates (3.3) with the last inequality and recalling that the weight $K$ is doubling, we get

$$
\begin{aligned}
\frac{1}{\int_{B_{\frac{R}{2}}} \frac{1}{K(x)} d x} \int_{B_{\frac{R}{2}}}|X u|^{2} d \nu & \leq \frac{C}{\int_{B_{\frac{R}{2}}} \frac{1}{K(x)} d x} \int_{B_{R}} \frac{\left|u-u_{R, \mu}\right|^{2}}{R^{2}} d \mu \\
& =C \frac{\int_{B_{R}} K(x) d x}{\int_{B_{\frac{R}{2}}} \frac{1}{K(x)} d x}\left(\frac{1}{\int_{B_{R}} K(x) d x} \int_{B_{R}} \frac{\left|u-u_{R, \mu}\right|^{2}}{R^{2}} d \mu\right) \\
& \leq C \frac{\int_{B_{R}} K(x) d x}{\int_{B_{\frac{R}{2}}} \frac{1}{K(x)} d x}\left(\frac{1}{\int_{B_{R}} \frac{1}{K(x)} d x} \int_{B_{R}}|X u|^{p_{0}} d \nu\right)^{\frac{2}{p_{0}}} \\
& \leq C_{1} \frac{f_{B_{\frac{R}{2}}} K(x) d x}{f_{B_{\frac{R}{2}}} \frac{1}{K(x)} d x}\left(\frac{1}{\int_{B_{R}} \frac{1}{K(x)} d x} \int_{B_{R}}|X u|^{p_{0}} d \nu\right)^{\frac{2}{p_{0}}} \\
& =C_{2}\left(\frac{1}{\int_{B_{R}} \frac{1}{K(x)} d x} \int_{B_{R}}|X u|^{p_{0}} d \nu\right)^{\frac{2}{p_{0}}}
\end{aligned}
$$

where

$$
C_{2}=C_{1} \frac{f_{B_{\frac{R}{2}}} K(x) d x}{f_{B_{\frac{R}{2}}} \frac{1}{K(x)} d x}
$$

Now observe that

$$
f_{B_{\frac{R}{2}}} K(x) d x \leq c \operatorname{essinf} \operatorname{Bin}_{\frac{R}{2}} \frac{1}{K} \quad \text { for all } B_{\frac{R}{2}} \subset \Omega
$$

thanks to the assumption that the pair $\left(K, \frac{1}{K}\right) \in A_{1}$. On the other hand, we obviously have

$$
\text { ess } \inf _{\frac{R}{2}} \frac{1}{K} \leq \frac{1}{K} \quad \text { a.e. in } B_{\frac{R}{2}}
$$

and therefore

$$
f_{B_{\frac{R}{2}}} K(x) d x \leq c f_{B_{\frac{R}{2}}} \frac{1}{K(x)} d x \text { for all } B_{\frac{R}{2}} \subset \Omega .
$$

It follows that the constant $C_{2}$ can be estimated by a constant independent of $B_{\frac{R}{2}}$, $\mu, \nu$. Now, from (3.5), applying Lemma 2.4 with $g=|X u|^{p_{0}}, q=\frac{2}{p_{0}}, f=0$ and $\vartheta=0$ we easily deduce the result.

\section{References}

[1] BJÖRN, A., and N. MAROLA: Moser iteration for (quasi)minimizers on metric spaces. Manuscripta Math. 121:3, 2006, 339-366.

[2] BoyARski, B.: Generalized solutions of a system of differential equation of the first order of elliptic type with discontinuos coefficients. - Sb. Math. 43, 1957, 451-503. 
[3] Capogna, L., D. Danielli, and N. Garofalo: An embedding theorem and the Harnack inequality for nonlinear subelliptic equations. - Comm. Partial Differential Equations 18:9-10, 1993, 1765-1794.

[4] Chanillo, S., and R. L. Wheeden: Weighted Poincaré and Sobolev inequalities and estimates for weighted Peano maximal functions. - Amer. J. Math. 107:5, 1985, 1191-1226.

[5] Cruz-Uribe, D., Sfo, K. Moen, and V. Naibo: Regularity of solutions to degenerate $p$ Laplacian equations. - J. Math. Anal. Appl. 401, 2013, 458-478.

[6] Cruz-Uribe, D. Sfo, P. Di Gironimo, and C. Sbordone: On the continuity of solutions to degenerate elliptic equations. - J. Differential Equations 250:6, 2011, 2671-2686.

[7] Di Fazio, G., and M. S. Fanciullo: Gradient estimates for elliptic systems in CarnotCarathéodory spaces. - Comment. Math. Univ. Carolin. 43:4, 2002, 605-618.

[8] Franchi, B., G. Lu, and R. L. Wheeden: Representation formulas and weighted Poincaré inequalities for Hörmander vector fields. - Ann. Inst. Fourier (Grenoble) 45:2, 1995, 577-604.

[9] García-Cuerva, J., and J. L. Rubio de Francia: Weighted norm inequalities and related topics. - North-Holland Math. Stud. 116, North-Holland Publishing Co., Amsterdam, 1985.

[10] Garofalo, N., and D. M. Nhieu: Isoperimetric and Sobolev inequalities for CarnotCarathéodory spaces and the existence of minimal surfaces. - Comm. Pure Appl. Math. 49, 1996, 1081-1144.

[11] GianazzA, U.: Higher integrability for quasiminima of functionals depending on vector fields. - Rend. Accad. Naz. Sci. XL Mem. Mat. (5) 17, 1993, 209-227.

[12] Giannetti, F.: Weak minima of integral functionals in Carnot-Carathéodory spaces. Ricerche Mat. 54:1, 2005, 255-270.

[13] Giannetti, F., and A. Passarelli di Napoli: Regularity results for minimizers of integral functionals with nonstandard growth in Carnot-Carathéodory spaces. - Rend. Lincei Mat. Appl. 21, 2010, 175-192.

[14] Giaquinta, M., and E. Giusti: On the regularity of the minima of variational integrals. Acta Math. 148, 1982, 31-46.

[15] Heinonen, J., T. KilpeläInen, and O. Martio: Nonlinear potential theory of degenerate elliptic equations. - Oxford Math. Monogr., 1993.

[16] Kinnunen, J., and N. Shanmugalingam: Regularity of quasi-minimizers on metric spaces. - Manuscripta Math. 105, 2001, 401-423.

[17] Lu, G.: Weighted Poincaré and Sobolev inequalities for vector fields satisfying Hörmander's condition and applications. - Rev. Mat. Iberoam. 8:3, 1992, 367-439.

[18] Maasalo, O. E., B. Stroffolini, and A. Verde: Local boundedness of minimizers of integral functionals with $(p, q)$-growth on metric spaces. - Funct. Approx. Comment. Math. 40:1, 2009, 127-138.

[19] Modica, G.: Quasminima of some degenerate functionals. - Ann. Mat. Pura Appl. (4) 142, 1986, 121-143.

[20] Moscariello, G.: Local boundedness of minimizers of certain degenerate functionals of the calculus of variations. - Nonlinear Anal. 23, 1994, 1587-1593.

[21] Nagel, A., E. M. Stein, and S. Waigner: Balls and metrics defined by vector fields I: Basic properties. - Acta. Math. 155:1, 1985, 103-147.

[22] Stromberg, J.-O., and A. Torchinsky: Weighted Hardy spaces. - Lecture Notes in Math. 1381, Springer-Verlag, Berlin, 1989.

[23] Torchinsky, A.: Real-variable methods in harmonic analysis. - Pure Appl. Math. 123, Academic Press, Inc., Orlando, FL, 1986.

[24] Zatorska-Goldstein, A.: Very weak solutions of nonlinear subelliptic equations. - Ann. Acad. Sci. Fenn. Math. 30, 2005, 407-436. 